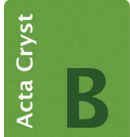

STRUCTURAL SCIENCE CRYSTAL ENGINEERING MATERIALS

ISSN 2052-5206

Received 23 March 2015

Accepted 24 March 2015

Keywords: transmission electron microscopy; powder diffraction; anion-deficient perovskites; modulated structures.

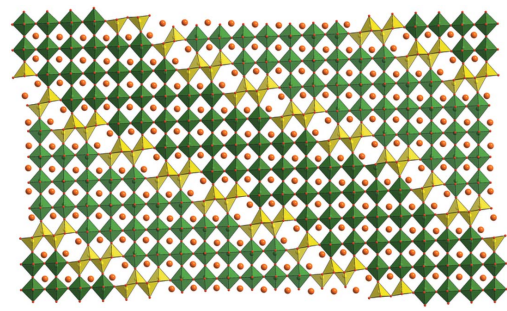

C 2015 International Union of Crystallography

\section{Taking a closer look for a broader view: combining powder diffraction with electron crystallography for a better understanding of modulated structures}

\author{
Lukáš Palatinus*
}

Department of Structure Analysis, Institute of Physics, Czech Academy of Sciences, Na Slovance 2, 18221 Prague, Czech Republic. *Correspondence e-mail: palat@fzu.cz

In the past few years, few fields of crystallography have gone through such dramatic development as electron crystallography. Over the last decade we have seen the advent of electron diffraction tomography (EDT), transforming electron diffraction into a tool routinely applicable (and used) for solution of unknown crystal structures, as well as the growth of aberration-corrected transmission electron microscopy, which allows the observation of crystal structures in an unprecedented degree of detail, with sub- $\AA$ resolution. Modern spectroscopic methods combined with the aberration correction can provide chemical information on the atomic scale. The result of all this progress is that electron crystallography gives answers to more and more questions that used to be the domain of X-ray or neutron diffraction, and is especially useful when the X-ray or neutron experiment needs to be performed on a powder material, which limits the diffraction information available.

The limitations of powder diffraction are particularly palpable when dealing with modulated structures. These structures are characterized by an average three-dimensional periodic structure and by a modulation function that is associated with every atom in the average unit cell and which describes how the average position of the atom is perturbed from one unit cell to another. This property leads to the occurrence of additional spots in the diffraction pattern called satellites (see van Smaalen, 2012, for an overview of the subject). Typically, one main reflection arising from the average structure is associated with a regular array of between two and ten satellite reflections. These satellites are usually weaker (sometimes much weaker) than the main reflections. Projecting such a complex diffraction pattern onto the one-dimensional $2 \theta$ axis of a powder diffraction pattern leads to a significant loss of information. As a result, indexing such a diffraction pattern without any prior information is extremely difficult or impossible. Even if this problem is solved, quite often with the aid of an electron diffraction experiment, the next task of finding the initial structural model is also extremely difficult. The modulation functions are continuous and need to be parameterized by certain well selected functions (typically a Fourier series or discontinuous step-like functions), and their choice is not obvious a priori. This means that not only is the structural model unknown in advance, so is the parameterization of the structural model - a situation fundamentally different from the analysis of periodic structures. While for single-crystal diffraction data of modulated structures methods exist for the solution of this problem (Hao et al., 1987; Palatinus, 2004), their applicability to powder diffraction data is very limited. However, once the structure model is known, state-of-theart powder diffraction data, ideally from synchrotron sources, are usually of sufficient quality for the Rietveld refinement of the structural model.

Batuk et al. (2015) demonstrate in their article how the tools of electron crystallography may be used to circumvent the limitations of powder diffraction and complement the structure analysis of modulated structures by powder diffraction. The authors combine the results of their previous research with new results to provide an impressive overview of the available methods and information they can provide. The material used for the demonstration is a series of anion-deficient perovskites, where the modulation is caused by an occurrence of crystallographic shear (CS) planes, which appear with an average periodicity that is generally incommensurate with the basic periodicity of the structure. This choice of material is particularly fortunate, because these structures 


\section{scientific commentaries}

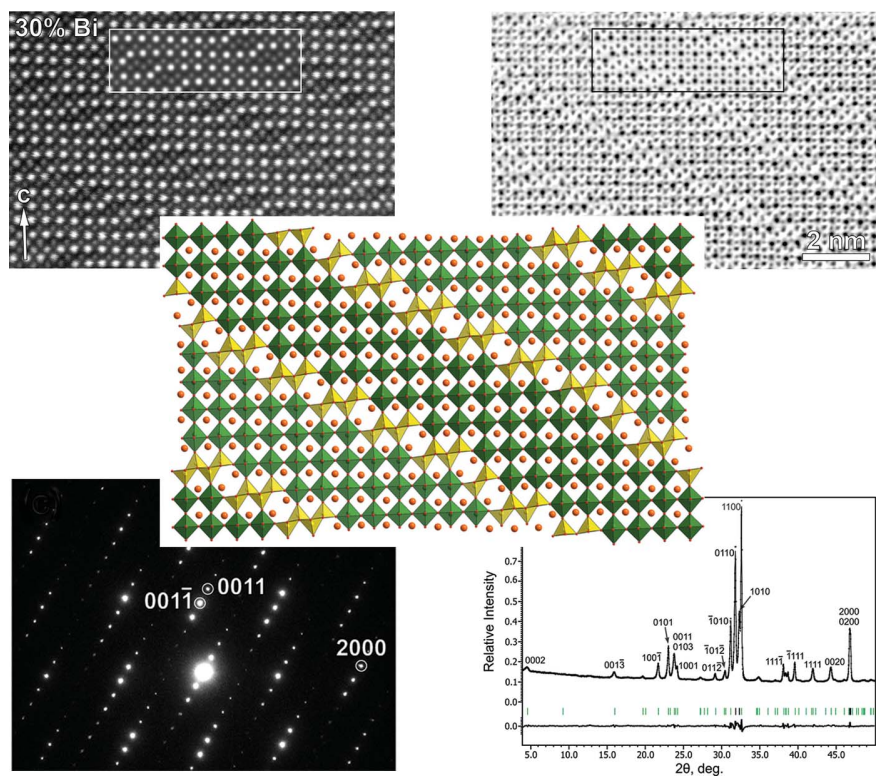

Figure 1

Combining electron microscopy methods with powder diffraction data provides a more accurate and more reliable structure model. Collage of images from Batuk et al. (2015).

exhibit a wide variety of features that complicate the structure analysis of modulated structures from powder patterns, including the generally large number of fairly strong satellites, large modulations described by discontinuous modulation functions and a combination of positional modulation with chemical ordering of atoms with similar scattering power. A special feature of the structures is that they have one short crystallographic axis, along which the atoms project as well defined columns, which is very favourable for the observation by atomic resolution transmission electron microscopy (TEM).

For a reader not versed in recent developments of the field the images are breathtaking. Three-dimensional EDT data allow for a direct and unambiguous determination of the lattice parameters and modulation vectors. Atomic resolution high-angle annular dark field (HAADF) images show perfectly the distribution of heavy atoms and of the orientation and type of CS planes. Annular bright field (ABF) images clearly show the position of oxygen columns and show the strong modulation of these columns. Energy-dispersive spectroscopy (EDS) analysis reveals the preference for $\mathrm{Pb}$ atoms over $\mathrm{Bi}$ atoms in the CS planes, while a detailed analysis of an electron-energy loss spectrum (EELS) at an $\mathrm{Fe} L_{2,3}$ edge allows an atomic resolution mapping of the coordination number of $\mathrm{Fe}$ across the structure.

Facing all these results, a question may cross our minds: can we expect electron crystallography to make X-ray or neutron powder diffraction obsolete in the near future? Without any doubt, the answer is negative for a number of reasons. Many materials are not stable under the electron beam, especially for high-resolution imaging. Electron microscopy also does not allow in situ experiments at high pressures or in complicated chemical environments. Not all structures provide sufficiently well defined projections for observation with atomic resolution techniques. But even for materials and experiments where these constraints do not apply, powder diffraction is a necessity because it gives representative information of the bulk material, while electron microscopy is a highly selective technique always carrying the danger of selecting a non-representative sample of the material; in most cases it is the average property of the bulk that is of interest. Finally, refinement against neutron or X-ray powder diffraction data still provides more accurate structural parameters than electron diffraction data. Some progress in this area has also recently been made for periodic structures, but its extension to modulated structures is not yet in sight. These advantages of neutron diffraction over electron diffraction or imaging are also well described in the paper by Batuk et al. (2015). Although most of the paper is focused on different TEM-based techniques, the final structural model used to interpret the details of the structure has been determined from the refinement of the neutron diffraction data (Fig. 1).

The article shows convincingly how the electron crystallography methods have grown to a rich source of detailed information on the crystal structures, and it should convince any reader that resorting to these methods may very quickly solve problems that seem intractable by the more traditional approaches. At the same time it is clear that electron microscopy alone is not enough to fully characterize a modulated structure and the key to success indeed lies in exploiting the complementarity and synergy between various methods.

\section{References}

Batuk, D., Batuk, M., Abakumov, A. M. \& Hadermann, J. (2015). Acta Cryst. B71, 127-143.

Hao, Q., Liu, Y. \& Fan, H. (1987). Acta Cryst. A43, 820-824.

Palatinus, L. (2004). Acta Cryst. A60, 604-610.

van Smaalen, S. (2012). Incommensurate Crystallography. Oxford University Press. 